\title{
Facile Synthesis Route of Au-Ag Nanostructures Soaked in PEG
}

\author{
Essy Kouadio Fodjo ${ }^{1,2 *}$, Ali Canlier ${ }^{2,3}$, Cong Kong4* , Ayhan Yurtsever ${ }^{5}$, \\ Pohan Lemeyonouin Aliou Guillaume ${ }^{6}$, Fato Tano Patrice ${ }^{7}$, Masayuki Abe ${ }^{5}$, \\ Tetsuya Tohei ${ }^{5}$, Akira Sakai ${ }^{5}$
}

\author{
${ }^{1}$ Laboratoire de Chimie Physique, Université Felix Houphouet-Boigny, Abidjan, Cote d'Ivoire \\ ${ }^{2}$ Department of Materials Science and Nanotechnology Engineering, Abdullah Gul University, Kayseri, Turkey \\ ${ }^{3}$ Department of Chemical Engineering and Applied Chemistry, College of Engineering, Chungnam National University, \\ Daejeon, Republic of Korea \\ ${ }^{4}$ Key Laboratory of East China Sea Fishery Resources Exploitation, Ministry of Agriculture, East China Sea Fisheries Research \\ Institute, Chinese Academy of Fishery Sciences, Shanghai, China \\ ${ }^{5}$ Graduate School of Engineering Science, Osaka University, Toyonaka, Osaka, Japan \\ ${ }^{6}$ UFR Sciences Biologiques, Peleforo-Gon-Coulibaly University, Korhogo, Cote d'Ivoire \\ ${ }^{7}$ Key Laboratory for Advanced Materials and Department of Chemistry, East China University of Science and Technology, \\ Shanghai, China \\ Email: ^kouadio.essy@univ-fhb.edu.ci, essykouadiofodjo@yahoo.fr, alicanlier@hotmail.com, ^kongcong@gmail.com, \\ ayhan.yurtsever@gmail.com, abe@stec.es.osaka-u.ac.jp, pohan.aliou@gmail.com, fatopatrice@yahoo.fr, \\ tohei@ee.es.osaka-u.ac.jp, sakai@ee.es.osaka-u.ac.jp
}

How to cite this paper: Fodjo, E.K., Canlier, A., Kong, C., Yurtsever, A., Guillaume, P.L.A., Patrice, F.T., Abe, M., Tohei, T. and Sakai, A. (2018) Facile Synthesis Route of $\mathrm{Au}-\mathrm{Ag}$ Nanostructures Soaked in PEG. Advances in Nanoparticles, 7, 37-45. https://doi.org/10.4236/anp.2018.72004

Received: April 5, 2018

Accepted: May 28, 2018

Published: May 31, 2018

Copyright $\odot 2018$ by authors and Scientific Research Publishing Inc. This work is licensed under the Creative Commons Attribution International License (CC BY 4.0).

http://creativecommons.org/licenses/by/4.0/

\begin{abstract}
A nanostructured gold-silver soaked in polyethylene glycol 400 (Au-Ag@PEG) is designed using gold(I) chloride and silver nitrate $\left(\mathrm{AgNO}_{3}\right)$ as precursors and, polyethylene glycol 400 (PEG) as capping agent. The result of the structure characterization using Selected Area Electron Diffraction (SAED) has showed that the synthesized nanomaterial has a good crystallinity while Transmission Electron Microscopy (TEM), energy dispersive X-ray spectrometry (EDX) and Dynamic Light Scattering (DLS) measurements suggest mixed Au-Ag nanoparticles with an average diameter size of around $7 \mathrm{~nm}$ and $30 \mathrm{~nm}$ for $\mathrm{Au}$ and $\mathrm{Ag}$ respectively.
\end{abstract}

\section{Keywords}

SAED Pattern, Au-Ag Nanomaterials Soaked in PEG, Nanoparticle Synthesis

\section{Introduction}

Since the intrinsic properties of the nanostructures are known to be determined 
by their size, shape and morphology, efforts have been devoted toward their design and synthesis [1] [2]. Among the various nanostructured materials, Au and Ag nanostructures have received particular interest. These nanomaterials have remarkable optical and antibacterial properties [3] [4] and, fascinating applications in wide areas such as sensing [5] [6] [7], catalysis [8], optical labeling [9] [10], drug delivery [11] [12], cancer diagnosis and its treatment [12] [13]. Moreover, to increase the above properties, the combination of two or more types of nanoparticles has been explored in different forms such as hybrid or core-shell with different morphologies [14] [15] [16]. Under a rational design, combining two or more metals into a single nanostructure will not only bridge the unique properties of individual materials to leverage research both fundamentally and practically, but will also improve conventional sensing, imaging and therapeutic efficacies [17]. Therefore, a variety of synthesis methods with diverse capping ligands such as alkanethiols, alkylamines, and organic polymers has been developed and is under investigation for eco-friendly and cost-effectiveness synthesis [18].

Commonly, gold nanoparticles (Au NPs) are synthesized using gold(III) $(\mathrm{Au}(\mathrm{III}))$ compounds as precursors [19]. This route using $\mathrm{Au}(\mathrm{III})$ undergoes several processing steps including gold(I) $(\mathrm{Au}(\mathrm{I}))$ before leading to the desired $\mathrm{Au}$ NPs. These different steps are the key to understand which catalyst should be used to fasten or slow down in order to obtain the required morphology and size for their further applications [1]. Although both $\mathrm{Au}(\mathrm{III})$ and $\mathrm{Au}(\mathrm{I})$ are common oxidation states of gold, precursors used in Au nanostructure synthesis are rarely based on $\mathrm{Au}(\mathrm{I})$ compounds. One of this unpopularity is related to the difficulties to handle $\mathrm{Au}(\mathrm{I})$ compounds. Compared with $\mathrm{Au}(\mathrm{III})$ compound, the solubility of $\mathrm{Au}(\mathrm{I})$ compound such as $\mathrm{AuCl}$ or $\mathrm{AuBr}$, is much lower in common solvent [20]. This low solubility prevents the $\mathrm{Au}(\mathrm{I})$ compound from being used in a conventional solution-phase synthesis. Besides, the standard reduction potential related to $\mathrm{Au}(\mathrm{I})(\mathrm{Au}+/ \mathrm{Au}=+1.68 \mathrm{~V})$ is relatively high [1]. This property gives another difficulty to handle with common reductant in which the reactivity with $\mathrm{Au}(\mathrm{I})$ undergoes fast reaction making difficult to control the morphology and the size of final Au NPs. Another crucial problem is that the $\mathrm{Au}(\mathrm{I})$ species tend to disproportionate to produce $\mathrm{Au}(0)$ and $\mathrm{Au}(\mathrm{III})$ species according to Equation (1): [1] [21]

$$
3 \mathrm{Au}(\mathrm{I}) \rightleftarrows 2 \mathrm{Au}(0)+\mathrm{Au}(\mathrm{III})
$$

These difficulties put some restrictions on the utilization of $\mathrm{Au}(\mathrm{I})$ compounds as reliable precursors for nanostructure synthesis. However, there are intrinsic merits for using $\mathrm{Au}(\mathrm{I})$ compounds as precursors. Some of the above-mentioned problems can be seen as huge advantages for the use of $\mathrm{Au}(\mathrm{I})$ precursor source: synthesis needs less energy (only one electron is needed to reduce $\mathrm{Au}(\mathrm{I})$ to $\mathrm{Au}(0)$ ), weak reductant can be used for Au NPs synthesis, and the synthesis can be done in short time [21]. These are a great deal to use an eco-friend catalyst. For these reasons, it is important to explore the use of $\mathrm{Au}(\mathrm{I})$-based precursors in 
the shape-controlled synthesis of $\mathrm{Au} / \mathrm{Ag}$ nanostructures.

In this study, we have synthesized a non-toxic Au@PEG and Ag-Au@PEG nanomaterials. The structure characterization using SAED and EDX has permitted to confirm the good crystallinity and composition of Au-Ag while TEM and DLS measurements suggested particle size of around $7 \mathrm{~nm}$ and $30 \mathrm{~nm}$ for $\mathrm{Au}$ and $\mathrm{Ag}$ respectively.

\section{Experimental Section}

\subsection{Reagents}

All reagents were of analytical grade and were used as received without any purification. Sodium citrate dehydrate $\left(\mathrm{HOC}(\mathrm{COONa})\left(\mathrm{CH}_{2} \mathrm{COONa}\right)_{2} \cdot 2 \mathrm{H}_{2} \mathrm{O}, 99 \%\right)$, silver nitrate $\left(\mathrm{AgNO}_{3}, \geq 99 \%\right)$ are from Sigma-Aldrich, polyethylene glycol 400 (PEG), Sodium tetrahydridoborate $\left(\mathrm{NaBH}_{4}, \geq 98.0 \%\right)$ and gold chloride $(\mathrm{AuCl}$, 99.9\%) are from Aldrich.

All solutions were prepared with deionized water (with $\rho 18.2 \mathrm{M} \Omega \mathrm{cm}$ ) obtained from a Mili-Q System (Millipore, USA).

\subsection{Au-Ag@PEG Preparation}

In Step 1 (Au@PEG synthesis), $73 \mathrm{mg}$ of $\mathrm{AuCl}$ is dissolved in $5 \mathrm{~mL}$ of PEG under vortex homogenization. After $5 \mathrm{~min}$ of homogenization; the mixture was centrifugated at $9000 \mathrm{rpm}$ for another $5 \mathrm{~min}$ to eliminate the Au microparticles. The obtained solution is mixed with a solution of sodium citrate $(42 \mathrm{mg}$ of sodium citrate dehydrate, previously dissolved in $25 \mathrm{~mL}$ DI water) under stirring (the final color is violet). The undergoing reaction is followed by recording for every hour, the UV-vis spectrum. In Step 2 (Ag NPs synthesis), $40 \mathrm{mg}$ of $\mathrm{AgNO}_{3}$ is dissolved in $15 \mathrm{~mL}$ of DI water, mixed with $2 \mathrm{~mL}$ of $\mathrm{NaBH}_{4}(6 \mathrm{mg})$ and left for $10 \mathrm{~min}$ of reaction [5]. Finally, the solutions obtained in Step 1 and that of Step 2 are mixed for the preparation of Au-Ag@PEG nanomaterials and, the UV-Vis is recorded every hour. After adding the mixture of Ag NPs, dark field is necessary to avoid the aggregation of $\mathrm{Ag}^{+}$and $\mathrm{Cl}^{-}$. All these reactions were done at room temperature $\left(20^{\circ} \mathrm{C}\right)$.

\subsection{For Crystal Structure Investigation}

The optical properties were measured using Ocean Optical 4000XR1 UV-Vis Spectrometer (USA) in the wavelength range of $200-1000 \mathrm{~nm}$. The structure, size, and composition of the NPs were characterized by transmission electron microscopy (TEM), scanning TEM equipped with a high angle annular dark-field detector (STEM-HAADF), Selected Area Electron Diffraction (SAED), and energy-dispersive X-ray spectroscopy (EDX) elemental mapping. SAED patterns and high-resolution TEM (HRTEM) images were acquired using a JEOL JEM-2100 instrument operated at an accelerating voltage of $200 \mathrm{kV}$. STEM-HAADF images and EDX elemental mapping of the NPs were obtained using a JEOL JEM-ARM200F transmission electron microscope operating at 200 
$\mathrm{kV}$. The samples for TEM measurements were prepared by placing a drop of the aqueous colloidal suspensions involving the synthesized NPs onto a carbon-coated copper-grid, and drying in vacuum overnight at room temperature. Image processing and analysis were carried out using the ImageJ software (https://imagej.net/Fiji).

\section{Results and Discussion}

\subsection{Optical Properties and Sizes of the Nanostructured Au-Ag@PEG}

To access the optical properties of the synthesized compound, the absorbance was followed during the synthesis process (Figure 1A and Figure 1B). Figure $1 \mathrm{~A}$ shows the UV-Vis spectra of Au@PEG while Figure 1B is related to Au-Ag@PEG. As displayed in Figure 1A, after two hours of synthesis, an obvious absorbance peak appears at around $530 \mathrm{~nm}$. This peak is the typical absorbance peak of $\mathrm{Au}$ [22] [23]. As the reaction continues, this absorbance peak becomes thinner, suggesting that the nanoparticles become more monodispersed. Furthermore, its intensity steadily increases to saturation at three hours. After three hours, all the graphs seem to overlap indicating that almost all the Au ions have been transformed to Au NPs. Therefore five hours is selected as the time for Au@PEG synthesis to make sure that the eventual remaining Au ions have been transformed.
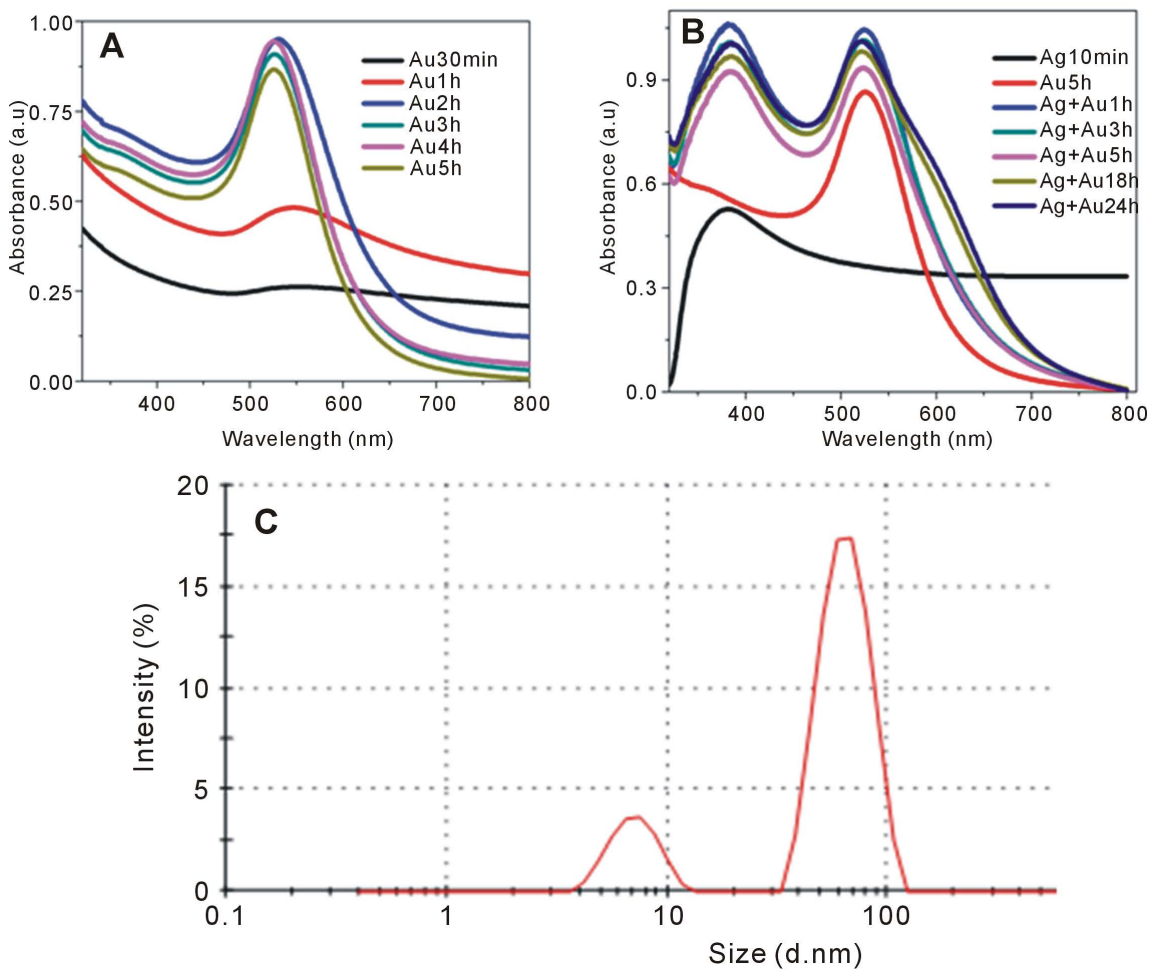

Figure 1. UV-vis-NIR absorption spectra of (A) Au@PEG, (B) Au-Ag@PEG recorded at different time intervals during the preparation of Au and Au-Ag@PEG NPs and (C) size distribution of the Au-Ag NPs heterostructures using DLS technique. 
In addition, the absorbance investigation of Au-Ag@PEG synthesized at different incubation times (Figure 1B) exhibit two absorbance peaks (one for Ag NPs at around $380 \mathrm{~nm}$ and the other for Au NPs at $530 \mathrm{~nm}$ ). No noticeable change is observed on the profile of the graph within $24 \mathrm{~h}$ of synthesis after mixing Au@PEG and Ag NPs. This result may suggest that Au NPs and Ag NPs individually coexist within $24 \mathrm{~h}$ in the mixture. Furthermore, additional experiments carried out using DLS technique (Figure 1C) show two peaks indication two different sizes with hydrodynamic diameter size ranging from $3 \mathrm{~nm}$ to 15 $\mathrm{nm}$ and from $30 \mathrm{~nm}$ to $130 \mathrm{~nm}$ with maximum at $7 \mathrm{~nm}$ and $60 \mathrm{~nm}$ respectively. This result suggests two types of size of nanoparticles.

\subsection{Structure Investigation of the Nanostructured Au-Ag@PEG}

The crystalline structures of synthesized materials were analyzed using SAED pattern (Figure 2A). The bright circular rings with bright spots suggest that the synthesized nanoparticles are highly crystalline nature [24] [25]. The observed crystalline nature of the NPs was further confirmed by the high-resolution transmission electron microscopy (HRTEM) lattice spacing (Figures 2B-2D), thus indicating different lattice planes of the Au-Ag NPs.

In order to find the lattice structure of the synthesized material, the determination of $\mathrm{d}$-spacing has been done using HRTEM images and SAED results. The different results indicate that both Ag NPs and Au NPs have fcc lattice. To provide further insights into the morphology and the elemental composition of the synthesized NPs, STEM-HAADF and EDX measurements were carried
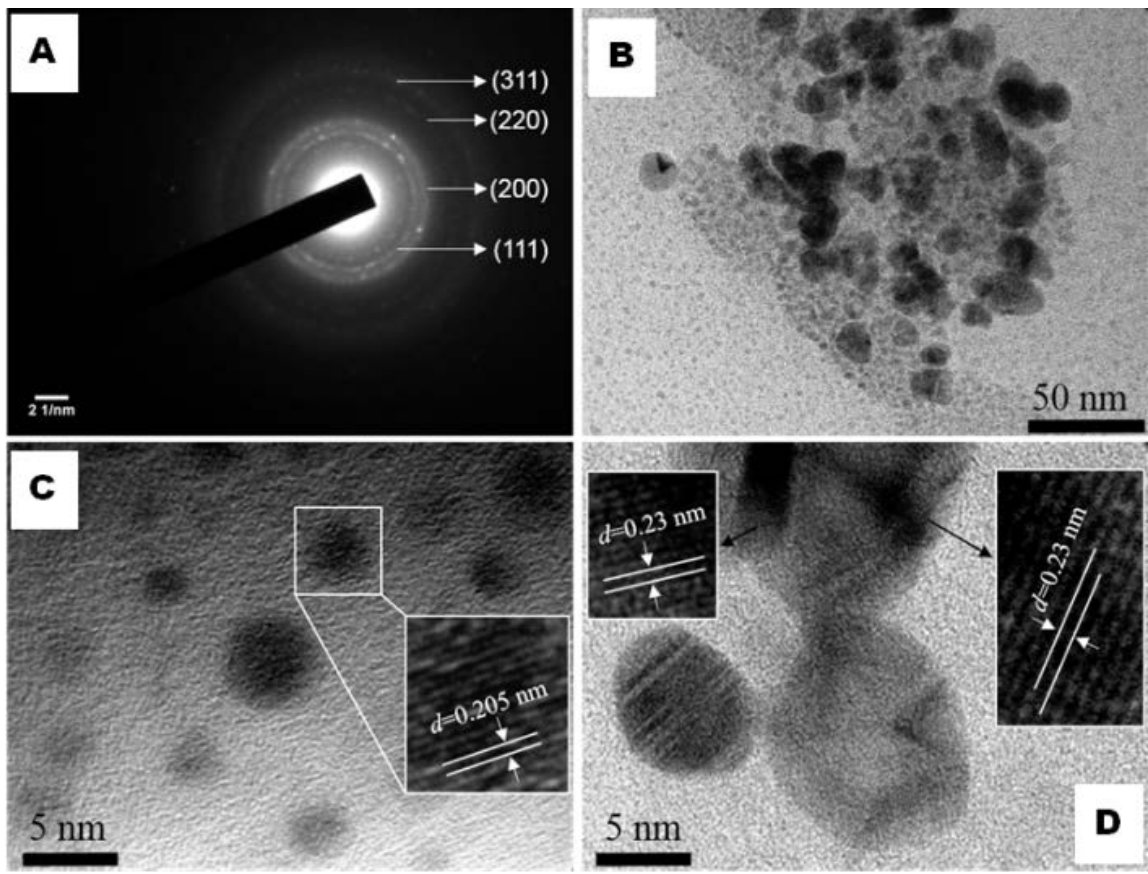

Figure 2. (A) SAED pattern, (B) TEM overview image of the synthesized NPs, displaying two types of NPs, (C) HRTEM image of the small size NPs and (D) HRTEM image of the larger size NPs, showing the inter-planar spacing (inset). 
out respectively (Figure 3 ). As can be seen in Figure $3 \mathrm{~A}$ and Figure 3B, two disparate types of NPs can be observed. Some small nanoparticles are deposited on the surface of the relatively large nanoparticles with average diameter size of $7 \mathrm{~nm}$ and $30 \mathrm{~nm}$ (HRTEM images) respectively confirming the existence of two types of morphology as suggested in DLS measurements. However, it is worth to note that the sizes of the nanostructure measured by DLS are much larger than those observed in STEM-HAADF or HRTEM images. This discrepancy in diameter size may be attributed to the steric hindrance effect of PEG. As PEG is a flexible linear polymer, it can dramatically influence the Brownian motion of particles by introducing additional frictional drag and thus producing larger size in the case of DLS measurements [26]. Indeed, the DLS technique can provide a full physical characterization of colloidal dispersions while STEM-HAADF or HRTEM requires the preparation of a dried nanoparticle or induces shrinkage of a dried nanoparticle specimen [19]. Consequently, this last characterization technique does not allow the inspection of the dimensions of the nanoparticles as they exist in this PEG solution. Moreover, according to the EDX results (Figure $3 \mathrm{C}$ ), the synthesized nanomaterial is mainly constituted of $\mathrm{Au}$ and $\mathrm{Ag}$ confirming the coexistence of the two types of nanoparticles as mentioned in above results (other peaks are substrate peaks). However, the peak intensities associated with $\mathrm{Ag}$ are relatively higher than those of $\mathrm{Au}$, indicating that the amount of $\mathrm{Ag}$ is higher than that of Au. To attribute the morphology to each type of nanoparticles, additional EDX investigation has been done (Figures 3D-3F). Figure 3D and Figure $3 \mathrm{E}$ show EDX elemental mapping images of individual $\mathrm{Ag}$ and $\mathrm{Au}$ respectively while Figure $3 \mathrm{~F}$ shows the overlay of these two nanoparticles.
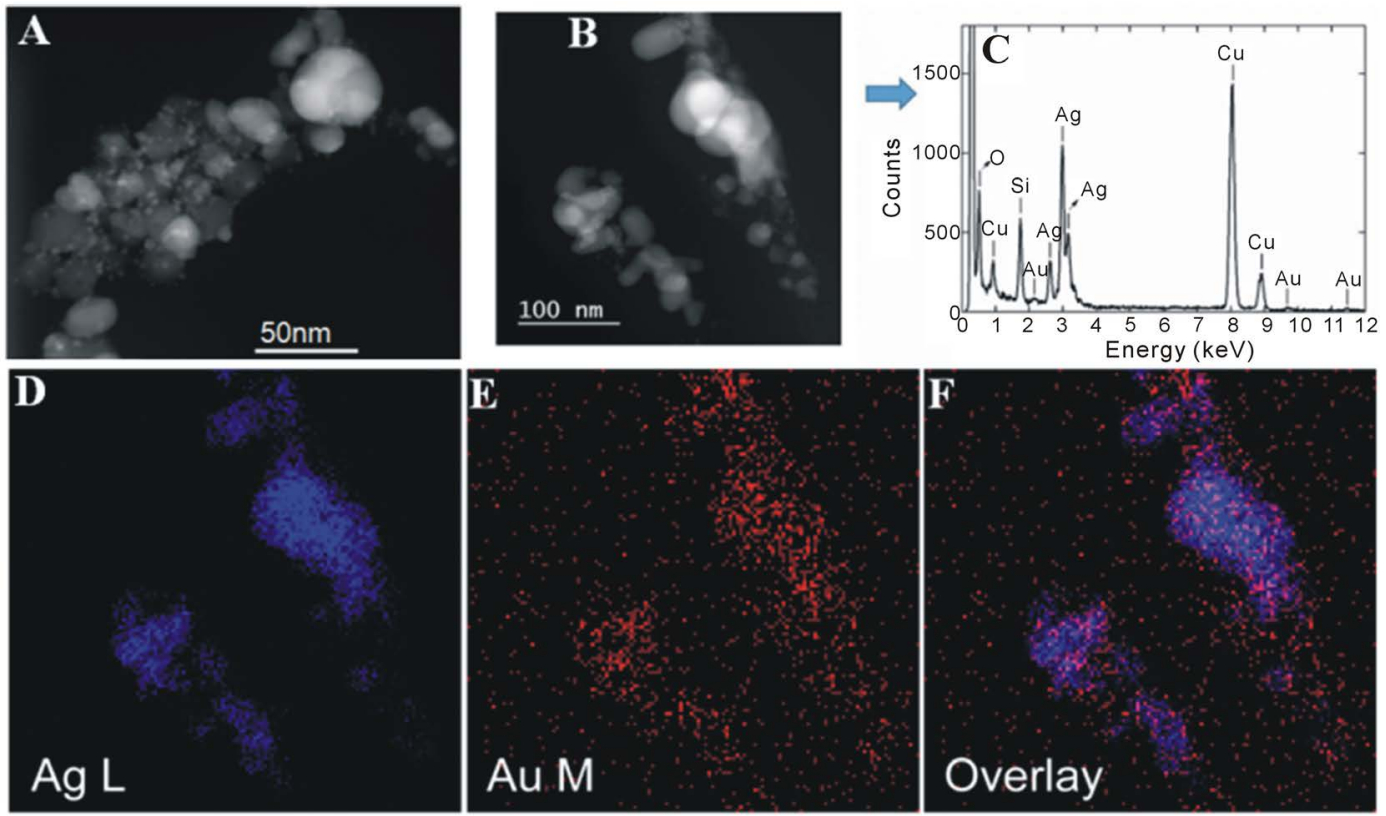

Figure 3. (A-B) STEM-HAADF images of as-synthesized nanomaterials, (C) a typical EDX spectrum and, EDX elemental mapping images of (D) Ag L, (E) Au M, and (F) an overlay of the Ag L and Au M. All the EDX measurements are collected from (B). 
As displayed, these images show clearly that the larger nanoparticles are Ag while the smaller ones are Au NPs. This result suggests that the average diameter size of $\mathrm{Au}$ NPs and Ag NPs are $7 \mathrm{~nm}$ and $30 \mathrm{~nm}$ respectively.

\section{Conclusion}

We have developed an eco-friendly method to synthesize an $\mathrm{Ag}$ decorated $\mathrm{Au}$ soaked in polyethylene glycol (Au-Ag@PEG) nanomaterials with diameter size around $7 \mathrm{~nm}$ and $30 \mathrm{~nm}$ for $\mathrm{Au}$ and $\mathrm{Ag}$ respectively. This rational design of Au-Ag@PEG nanomaterials combines two metals into a single nanostructure. As Au NPs and Ag NPs soaked in PEG are known to have potential medical application that directly comes in contact with the human body, this nanomaterial will not only bridge unique properties of individual materials, but may also improve conventional therapeutic efficacies besides their application in various products such as cosmetics, foods and consumer goods.

\section{Acknowledgements}

This work was supported by The World Academic of Science (TWAS) under Grant No. 16-510 RG/CHE/AF/AC_G-FR3240293301, The Scientific and Technological Research Council of Turkey (TUBITAK, 2221-Fellowships for Visiting Scientists and Scientists on Sabbatical Leave), and Grant-in-Aid for Scientific Research (JP17H03236) from the Ministry of Education, Culture, Sports, Science and Technology of Japan (MEXT). C. K. thanks the National Natural Science Foundation of China (31701698). A part of the present experiments was carried out by using a facility in the Research Center for Ultra-High Voltage Electron Microscopy, Osaka University.

\section{References}

[1] Zeng, J., Ma, Y., Jeong, U. and Xia, Y. (2010) $\mathrm{Au}^{\mathrm{I}}$ : An Alternative and Potentially Better Precursor than $\mathrm{Au}^{\mathrm{III}}$ for the Synthesis of $\mathrm{Au}$ Nanostructures. Journal of Materials Chemistry, 20, 2290-2301. https://doi.org/10.1039/b922571d

[2] Riaz, S., Qu, L., Fodjo, E.K., Ma, W. and Long, Y.-T. (2014) Thioanisole Induced Size-Selective Fragmentation of Gold Nanoparticles. RSC Advances, 4, 14031-14034. https://doi.org/10.1039/C4RA00528G

[3] Shankar, S., Jaiswal, L., Aparna, R.S.L. and Prasad, R.G.S.V. (2014) Synthesis, Characterization, in Vitro Biocompatibility, and Antimicrobial Activity of Gold, Silver and Gold Silver Alloy Nanoparticles Prepared from Lansium domesticum Fruit Peel Extract. Materials Letters, 137, 75-78. https://doi.org/10.1016/j.matlet.2014.08.122

[4] Lee, E.-J., Chang, M.-H., Kim, Y.-S. and Kim, J.-Y. (2013) High-Pressure Polyol Synthesis of Ultrathin Silver Nanowires: Electrical and Optical Properties. APL Materials, 1, Article ID: 042118. https://doi.org/10.1063/1.4826154

[5] Fodjo, E.K., Riaz, S., Li, D.-W., Qu, L.-L., Marius, N.P., Albert, T. and Long, Y.-T. (2012) Cu@Ag/ $\beta-\mathrm{AgVO}_{3}$ as a SERS Substrate for the Trace Level Detection of Carbamate Pesticides. Analytical Methods, 4, 3785-3791. https://doi.org/10.1039/c2ay25635e

[6] Elshawy, O.E., Helmy, E.A. and Rashed, L.A. (2016) Preparation, Characterization 
and in Vitro Evaluation of the Antitumor Activity of the Biologically Synthesized Silver Nanoparticles. Advances in Nanoparticles, 5, 149. https://doi.org/10.4236/anp.2016.52017

[7] La Spada, L., Iovine, R. and Vegni, L. (2012) Nanoparticle Electromagnetic Properties for Sensing Applications. Advances in Nanoparticles, 1, 9.

https://doi.org/10.4236/anp.2012.12002

[8] Sandoval, A., Aguilar, A., Louis, C., Traverse, A. and Zanella, R. (2011) Bimetallic $\mathrm{Au}-\mathrm{Ag} / \mathrm{TiO}_{2}$ Catalyst Prepared by Deposition-Precipitation: High Activity and Stability in CO Oxidation. Journal of Catalysis, 281, 40-49. https://doi.org/10.1016/j.jcat.2011.04.003

[9] Kretschmer, F., Mühlig, S., Hoeppener, S., Winter, A., Hager, M.D., Rockstuhl, C., Pertsch, T. and Schubert, U.S. (2014) Survey of Plasmonic Nanoparticles: From Synthesis to Application. Particle \& Particle Systems Characterization, 31, 721-744. https://doi.org/10.1002/ppsc.201300309

[10] Kaydashev, V., Ferrari, P., Heard, C., Janssens, E., Johnston, R.L. and Lievens, P. (2016) Optical Absorption of Small Palladium-Doped Gold Clusters. Particle \& Particle Systems Characterization, 33, 364-372.

https://doi.org/10.1002/ppsc.201600036

[11] Attia, Y.A., Altalhi, T.A. and Gobouri, A.A. (2015) Thermal Stability and Hot Carrier Dynamics of Gold Nanoparticles of Different Shapes. Advances in Nanoparticles, 4, 85. https://doi.org/10.4236/anp.2015.44010

[12] Jackson, T.C., Patani, B.O. and Ekpa, D.E. (2017) Nanotechnology in Diagnosis: A Review. Advances in Nanoparticles, 6, 93. https://doi.org/10.4236/anp.2017.63008

[13] Daniel, M.-C. and Astruc, D. (2004) Gold Nanoparticles: Assembly, Supramolecular Chemistry, Quantum-Size-Related Properties, and Applications toward Biology, Catalysis, and Nanotechnology. Chemical Reviews, 104, 293-346. https://doi.org/10.1021/cr030698+

[14] Subramanian, V., Wolf, E.E. and Kamat, P.V. (2004) Catalysis with $\mathrm{TiO}_{2} / \mathrm{Gold} \mathrm{Na-}$ nocomposites. Effect of Metal Particle Size on the Fermi Level Equilibration. Journal of the American Chemical Society, 126, 4943-4950. https://doi.org/10.1021/ja0315199

[15] Cozzoli, P.D., Fanizza, E., Comparelli, R., Curri, M.L., Agostiano, A. and Laub, D. (2004) Role of Metal Nanoparticles in $\mathrm{TiO}_{2} / \mathrm{Ag}$ Nanocomposite-Based Microheterogeneous Photocatalysis. The Journal of Physical Chemistry B, 108, 9623-9630. https://doi.org/10.1021/jp0379751

[16] Khan, M.M., Ansari, S.A., Amal, M.I., Lee, J. and Cho, M.H. (2013) Highly Visible Light Active Ag@TiO 2 Nanocomposites Synthesized Using an Electrochemically Active Biofilm: A Novel Biogenic Approach. Nanoscale, 5, 4427-4435. https://doi.org/10.1039/c3nr00613a

[17] Chen, P.-C., Roy, P., Chen, L.-Y., Ravindranath, R. and Chang, H.-T. (2014) Gold and Silver Nanomaterial-Based Optical Sensing Systems. Particle \& Particle Systems Characterization, 31, 917-942. https://doi.org/10.1002/ppsc.201400043

[18] Samal, A.K., Polavarapu, L., Rodal-Cedeira, S. and Liz-Marzan, L.M. (2013) Size Tunable Au@Ag Core-Shell Nanoparticles: Synthesis and Surface-Enhanced Raman Scattering Properties. Langmuir, 29, 15076-15082. https://doi.org/10.1021/la403707j

[19] Rahme, K., Chen, L., Hobbs, R.G., Morris, M.A., O’Driscoll, C. and Holmes, J.D. (2013) PEGylated Gold Nanoparticles: Polymer Quantification as a Function of PEG Lengths and Nanoparticle Dimensions. RSC Advances, 3, 6085-6094. https://doi.org/10.1039/C3RA22739A 
[20] Wolley, E.D. (1969) Process for Diffusing Gold into Semiconductor Material. Google Patents.

[21] Lu, X., Tuan, H.Y., Korgel, B.A. and Xia, Y. (2008) Facile Synthesis of Gold Nanoparticles with Narrow Size Distribution by Using $\mathrm{AuCl}$ or $\mathrm{AuBr}$ as the Precursor. Chemistry-A European Journal, 14, 1584-1591.

https://doi.org/10.1002/chem.200701570

[22] Wu, W., Shen, J., Banerjee, P. and Zhou, S. (2011) A Multifuntional Nanoplatform Based on Responsive Fluorescent Plasmonic ZnO-Au@PEG Hybrid Nanogels. Advanced Functional Materials, 21, 2830-2839.

https://doi.org/10.1002/adfm.201100201

[23] Kong, C., Wang, Y., Fodjo, E.K., Yang, G.-X., Han, F. and Shen, X.-S. (2017) Loop-Mediated Isothermal Amplification for Visual Detection of Vibrio parahaemolyticus Using Gold Nanoparticles. Microchimica Acta, 185, 35.

https://doi.org/10.1007/s00604-017-2594-4

[24] Philip, D. (2010) Green Synthesis of Gold and Silver Nanoparticles Using Hibiscus rosa sinensis. Physica E: Low-Dimensional Systems and Nanostructures, 42, 1417-1424. https://doi.org/10.1016/j.physe.2009.11.081

[25] Amali, A.J., Aranishi, K., Uchida, T. and Xu, Q. (2013) PdPt Nanocubes: A High-Performance Catalyst for Hydrolytic Dehydrogenation of Ammonia Borane. Particle \& Particle Systems Characterization, 30, 888-892. https://doi.org/10.1002/ppsc.201300100

[26] Sun, L., Zhang, H., An, W., Hao, A. and Hao, J. (2010) Vesicles Prepared by $\beta$-Cyclodextrins Inclusion Complexes Based on Switching Supramolecular Interaction Models Induced by Mixed Solvents. Journal of Inclusion Phenomena and Macrocyclic Chemistry, 68, 277-285. https://doi.org/10.1007/s10847-010-9785-5 